\title{
Primary Ewing's sarcoma of the sinonasal tract, eroding the ethmoid and sphenoid sinus with intracranial extension: A rare case report
}

\author{
MARIA EMANUELA NEGRU ${ }^{1}$, ANDREA PIETRO SPONGHINI ${ }^{1}$, DAVID RONDONOTTI ${ }^{1}$, \\ FRANCESCA PLATINI ${ }^{1}$, MARCO GIAVARRA ${ }^{1}$, LAURA FORTI ${ }^{1}$, MARIANGELA LOMBARDI ${ }^{2}$, \\ LAURA MASINI $^{3}$, RENZO BOLDORINI $^{4}$ and ALESSANDRA GALETTO ${ }^{1}$
}

\author{
${ }^{1}$ Medical Oncology Unit, ${ }^{2}$ Radiodiagnostic and Interventional Radiology Institute, ${ }^{3}$ Department of Radiotherapy, \\ ${ }^{4}$ Department of Health Sciences, School of Medicine, University Hospital Maggiore della Carità, I-28100 Novara, Italy
}

Received February 26, 2015; Accepted March 5, 2015

DOI: $10.3892 / \mathrm{mco} .2015 .548$

\begin{abstract}
Ewing's sarcoma (ES) is an aggressive tumour that may present with skeletal and extraskeletal forms. The extraskeletal form is rarely encountered in the head and neck region and is extremely rare in the sinonasal tract. This is the case report of a ES of the ethmoid sinus with intracranial and orbital extension in a 33-year-old male patient who presented with anosmia, epistaxis, reduction of visual acuity in the left eye and headache. On otorhinolaryngological clinical examination and biopsy via flexible endoscope, the lesion was misdiagnosed as ethmoid sinus carcinoma. The subsequent magnetic resonance imaging (MRI) of the brain revealed a large mass $(6 \times 7 \mathrm{~cm})$ eroding the ethmoid and sphenoid sinuses, extending beyond the orbits and occupying the anterior cranial fossa with a maximum extension of $\sim 5 \mathrm{~cm}$. The patient underwent surgical resection and the microscopic examination of the specimen established the diagnosis of ES (immunohistochemically positive for CD99, neuron-specific enolase, CD56, synaptophysin, pancytokeratin, low-molecular weight cytokeratins and vimentin. The periodic acid Schiff stain exhibited strong intracytoplasmic block positivity and fluorescence in situ hybridization revealed a t $(22 ; 11)$ translocation. First-line chemotherapy was administered for 3 cycles; however, on restaging MRI, local disease progression was diagnosed. The patient received radiotherapy and second-line chemotherapy for 6 cycles. At 15 months after the diagnosis, the patient remains recurrence-free and maintains a good functional status and quality of life.
\end{abstract}

Correspondence to: Dr Andrea Pietro Sponghini, Medical Oncology Unit, University Hospital Maggiore della Carità, 18 Corso Mazzini, I-28100 Novara, Italy

E-mail: asponghini@libero.it

Key words: Ewing's sarcoma, sinonasal tract, intracranial extension, magnetic resonance imaging, chemotherapy

\section{Introduction}

Ewing's sarcoma (ES) is an aggressive tumour with a high incidence of local recurrence and distant metastasis, which is more common in men compared with women, particularly in the first 2-3 decades of life (1). ES may present with skeletal and extraskeletal forms. The skeletal form is more common and typically occurs in the long bones of the extremities. The extraskeletal form occurs in the soft tissues of the lower extremities, paravertebral tissues, chest wall, retroperitoneum and rarely in the head and neck region. ES of the sinonasal tract is a rare occurrence and the number of clinical studies published in the world literature, describing clinical course and therapeutic approaches, is currently limited (2). Diagnosis is based on history, immunostaining with at least two neural markers, ultrastructural examination and evidence of an abnormal $t(22 ; 11)$ translocation, which is the hallmark of ES (1). This is the case report of a ES of the ethmoid sinus with intracranial and orbital extension in a 33-year-old male patient who presented with anosmia, epistaxis, reduction of visual acuity in the left eye and headache.

\section{Case report}

On August, 2013, a 33-year-old man underwent an otorhinolaryngological clinical examination due to a history of anosmia, epistaxis, reduction of visual acuity in the left eye and headache. An otorhinolaryngological biopsy via flexible endoscope was performed and an ethmoid sinus carcinoma was diagnosed. The patient subsequently underwent a magnetic resonance imaging (MRI) scan of the brain, which revealed a large mass $(6 \times 7 \mathrm{~cm})$ eroding the ethmoid and sphenoid sinuses, infiltrating the upper portion of the nasal septum and extending to the upper posterior region of the nasal cavities, affecting the inferior turbinates, particularly the right turbinate. The lesion extended beyond the orbits, coming into contact with the upper and medial rectus muscles on the right side and with the medial rectus muscle on the left side. The lesion also incorporated the intracranial portion of the optic nerves. The pathological tissue occupied the anterior cranial fossa with a 

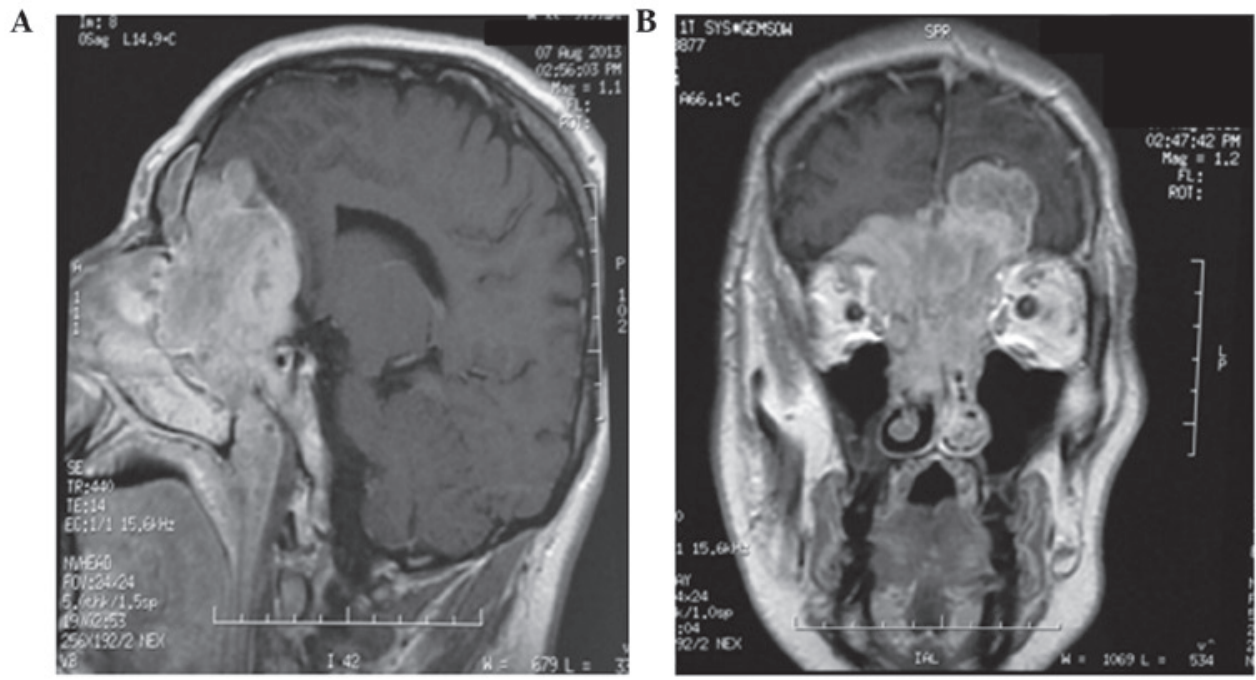

Figure 1. Preoperative magnetic resonance imaging, (A) sagittal and (B) coronal, spin-echo T1-weighted images, following intravenous gadolinium administration. The sequences revealed the presence of solid tissue with inhomogeneous enhancement eroding the ethmoid and sphenoid sinuses, infiltrating the upper portion of the nasal septum and extending to the medial wall of the orbits, displacing and compressing the frontal lobes.

maximum extension of $\sim 5 \mathrm{~cm}$, displacing and compressing the frontal lobes, as well as the genu and horns of the corpus callosum (Fig. 1). Subsequently, the patient underwent surgical (combined neurosurgical and otorhinolaryngological) removal of the lesion. The microscopic examination of the specimen revealed a population of epithelioid cells with round nuclei and scant cytoplasm, with positive resection margins (R2). A high number of mitotic figures and focal necrosis were observed. The tumour cells were immunohistochemically positive for CD99, neuron-specific enolase, CD56 and synaptophysin and also for pancytokeratin, low-molecular weight cytokeratins and vimentin. The periodic acid Schiff (PAS) stain exhibited strong intracytoplasmic block positivity (Fig. 2). Furthermore, interphase fluorescence in situ hybridization (FISH) revealed a $\mathrm{t}(22 ; 11)$ translocation. Subsequently, the diagnosis of ES was established based on the histopathology, immunoprofile and FISH results. The diagnosis was also confirmed following review of the slides by Professor J. Rosai on September, 2013.

The first oncological clinical examination was performed at the end of September, 2013.

A chemotherapy protocol that consisted of doxorubicin $\left(25 \mathrm{mg} / \mathrm{m}^{2}\right.$ on days $\left.1-3\right)$, ifosfamide $\left(3,000 \mathrm{mg} / \mathrm{m}^{2}\right.$ on days 1-3), 2-mercaptoethane sulfonate sodium (mesna; $3,000 \mathrm{mg} / \mathrm{m}^{2}$ on days $1-3$ ), vincristine (2 mg on day 1) and granulocyte colony-stimulating factor (G-CSF) for prophylaxis, was administered for a total of 3 cycles. On restaging computed tomography, at the site of intervention, a hypervascular inhomogeneous area sized 4,7x2,7 cm was identified, confirmed on the subsequent MRI as local disease progression. Therefore, the patient was ultimately treated with radiotherapy and second-line chemotherapy. A total radiation dose of 54 Gy was delivered to the naso-ethmoid sinus region. The second-line chemotherapy consisted of cyclophosphamide $\left(1,200 \mathrm{mg} / \mathrm{m}^{2}\right.$ on day 1$)$, etoposide $\left(150 \mathrm{mg} / \mathrm{m}^{2}\right.$ on days $\left.1-3\right)$, mesna $\left(2,000 \mathrm{mg} / \mathrm{m}^{2}\right.$ on day 1$)$ and prophylactic G-CSF administered for 6 cycles. The full treatment was administered every 21 days over the course of 9 months. The patient experience certain treatment-related toxicities, including neutropenic fever (grade 4, according to the Common Toxicity Criteria, v3.0; accessed at http://ctep.cancer.gov/protocol Development/electronic_applications/docs/ctcaev3.pdf) that required hospitalization. At 15 months after the diagnosis, the patient remains recurrence-free (Fig. 3) and maintains a good functional status and quality of life.

\section{Discussion}

We herein report a rare localization of ES in a young male patient, who recovered well and currently remains recurrence-free.

ES was first described by Ewing, an American pathologist, in 1921 (3). ES is an unusual disease, comprising 4-6\% of all primary bone tumours (4). Involvement of the head and neck in ES is unusual, accounting for $\sim 1-4 \%$ of all cases, with primary ES originating from the sinonasal tract being extremely rare. ES is more often diagnosed during the second and third decades of life, with a male gender predominance (4).

Microscopically, these tumours are composed of uniform small round cells with round nuclei containing fine chromatin, scanty clear or eosinophilic cytoplasm and PAS-positive intracytoplasmic glycogen granules. In the sinonasal tract, the differential diagnosis includes all tumours composed of small round cells, such as olfactory neuroblastoma and undifferentiated carcinoma (5). The diagnosis requires a histopathological examination, immunohistochemistry and cytogenetics. ES is characterized by translocation of the Ewing's sarcoma gene (EWS) gene, which is located on 22q12. $E W S$ is fused with the friend leukemia virus integration site 1 gene (FLI-1), which is located on 11q24, resulting in a $\mathrm{t}(22 ; 11)$ translocation that is present in $85 \%$ of the cases of ES and in $>90 \%$ of all extraosseous ESs (6). A FISH test is used to detect the EWS-FLI-1 fusion gene. The combination of the histological, molecular and genetic characteristics establishes the diagnosis of ES. This diagnosis is possible only following complete histopathological examination of the surgical specimen; in fact, during the first otorhinolaryngological clinical 
A

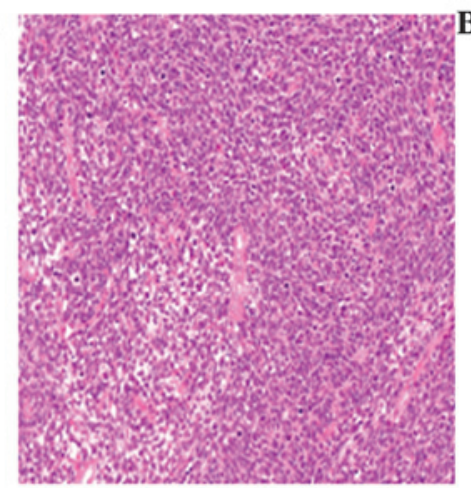

C

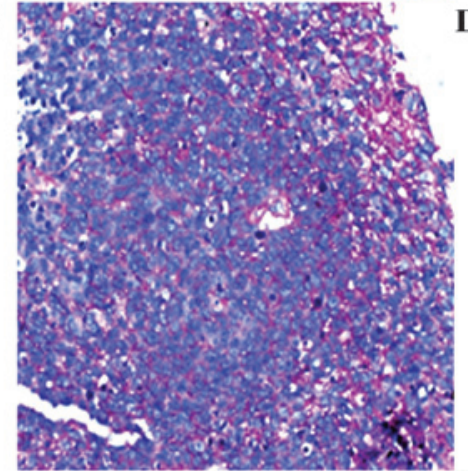

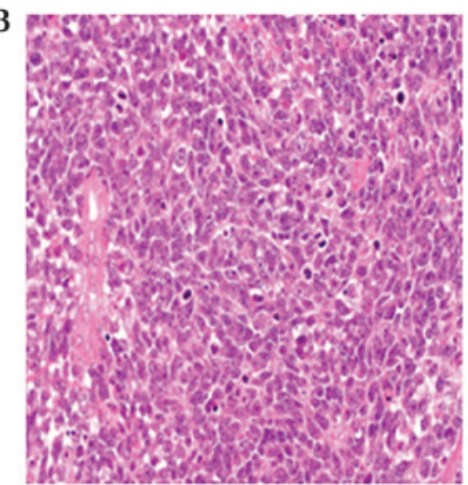

D.8.

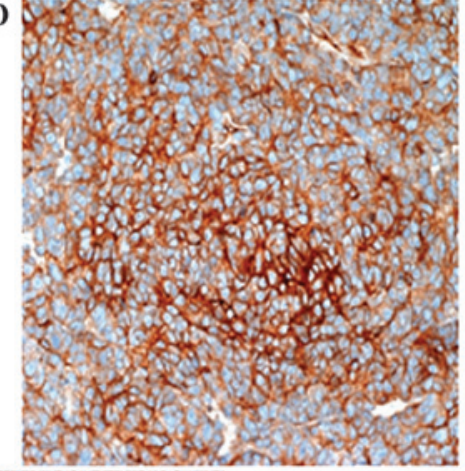

$\mathbf{E}$

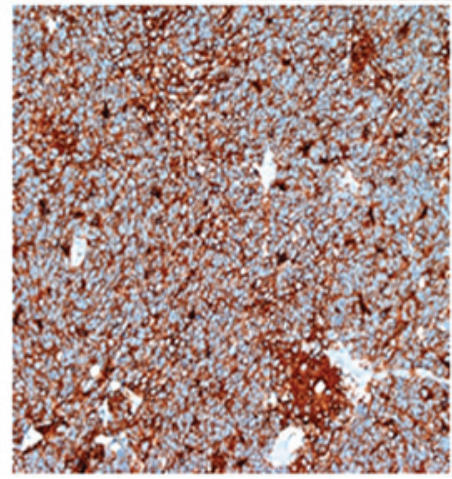

Figure 2. Immunostaining of tumour samples. The tumour is composed of broad sheets of small round cells with scant clear cytoplasm and coarse chromatin. Hematoxylin and eosin staining, magnification (A) x200; (B) x400. (C) The cytoplasm is typically periodic acid Schiff-positive, as it contains glycogen (magnification, x400). The tumour has a high mitotic index and the tumour cells are diffusely positive for (D) CD99 (magnification, x400) and (E) low-molecular weight cytokeratin (immunoperoxidase staining; magnification, x200).
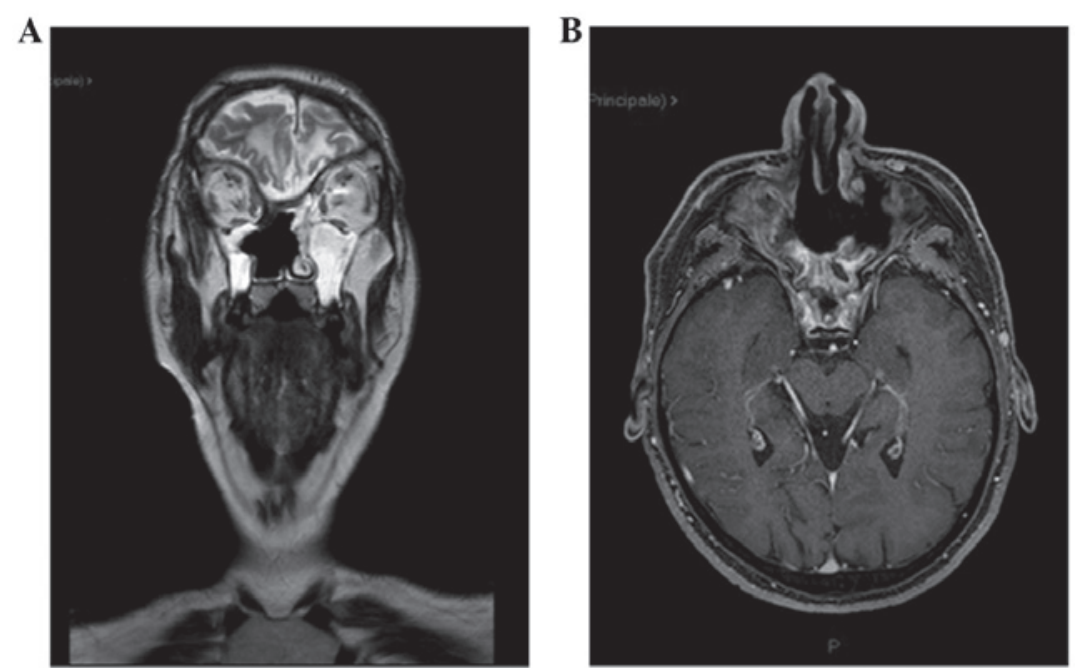

Figure 3. Postoperative magnetic resonance imaging. (A) Turbo spin-echo T2-weighted coronal sequence 11 months after the diagnosis and (B) T1-weighted axial sequence 14 months after the diagnosis. The images show the surgical cavity with inflammatory thickening and no signs of recurrence; (B) also shows persistent reduced inflammatory thickening, with no signs of recurrence. 
examination, the lesion was misdiagnosed as ethmoid sinus carcinoma.

When treating ES, a multidisciplinary approach consisting of surgical resection and radiotherapy plus chemotherapy has increased the 5-year survival rate from 20 to $58 \%(7,8)$. The most effective chemotherapy agents against ES are vincristine, doxorubicin, cyclophosphamide, ifosfamide and etoposide. Using the strategy of interval of chemotherapy cycles, the Children's Oncology Group has demonstrated a 5-year event-free survival of $73 \%$ in patients with localized tumours treated with these five drugs (9). The majority of the patients exhibit a rapid response of their primary tumour site following chemotherapy initiation. However, recurrence of resistant tumours either during or after completion of therapy occurs in up to one-third of the patients, constituting a significant problem (10). In the present case, the patient had a recurrence after 3 cycles of first-line chemotherapy; however, following radiotherapy and 6 cycles of second-line chemotherapy, the patient recovered well without evidence of recurrent disease. At 15 months after diagnosis the patient maintains a good functional status and quality of life.

To the best of our knowledge, the availability of scientific literature regarding ES of the ethmoid sinus is limited; however, our results are in accordance with currently available literature data $(2,10)$.

\section{Acknowledgements}

The authors would like to thank Professor J. Rosai, MD for kindly revising the immunohistochemical slides.

\section{References}

1. Tanboon J, Sitthinamsuwan B, Paruang T, Marrano P and Thorner PS: Primary intracranial Ewing sarcoma with an unusually aggressive course: A case report and review of the literature. Neuropathology 32: 293-300, 2012.

2. Li M, Hoschar AP, Budd GT, Chao ST and Scharpf J: Primary Ewing's sarcoma of the ethmoid sinus with intracranial and orbital extension: Case report and literature review. Am J Otolaryngol 34: 563-568, 2013.

3. Ewing J: Diffuse endothelioma of bone. Pathol Soc 21: 17-24, 1921.

4. Balamuth NJ and Womer RB: Ewing's sarcoma. Lancet Oncol 11: 184-192, 2010.

5. Yeshvanth SK, Ninan K, Bhandary SK, Lakshinarayana KP, Shetty JK and Makannavar JH: Rare case of extraskeletal Ewings sarcoma of the sinonasal tract. J Cancer Res Ther 8: 142-144, 2012.

6. Delattre O, Zucman J, Melot T, et al: The Ewing family of tumors - a subgroup of small-round-cell tumors defined by specific chimeric transcripts. N Engl J Med 331: 294-299, 1994.

7. de Bree R, van der Waal I, de Bree E and Leemans CR: Management of adult soft tissue sarcomas of the head and neck. Oral Oncol 46: 786-790, 2010.

8. Whaley JT, Indelicato DJ, Morris CG, Hinerman RW, Amdur RJ, Mendenhall WM, Keole SR and Marcus RB Jr: Ewing tumors of the head and neck. Am J Clin Oncol 33: 321-326, 2010.

9. Womer RB, West DC, Krailo MD, et al: Randomized controlled trial of interval-compressed chemotherapy for the treatment of localized Ewing sarcoma: A report from the Children's Oncology Group. J Clin Oncol 30: 4148-4154, 2012.

10. Ahmed AA, Zia H and Wagner L: Therapy resistance mechanisms in Ewing's sarcoma family tumors. Cancer Chemother Pharmacol 73: 657-663, 2014. 